\title{
Quantitative Analysis of Psychological Endurance Capability in Basketball Sport
}

\author{
Huan Chu \\ Department of public course, Jiangxi Institute of Fashion Technology,Nanchang,332201, China
}

Key words: Basketball, Psychological endurance capability, Quantitative analysis.

\begin{abstract}
Basketball is a charming sport. Countless fans in the world are crazy about it. It is also one of main events of the largest sport contest - Olympic Games. The research shows in large-scale basketball competition, strength and technical level of each high-level team do not differ a lot. Competition result is closely related to athletes' psychological quality and actual performance. As basketball competition level increasingly improves and competitiveness intensifies, how to let athletes have strong psychological endurance capability becomes a key problem.
\end{abstract}

\section{Introduction}

Generally speaking, the process of basketball sport event is very long, and athletes have large physical output. In such case, members of both teams will not differ a lot in strength and physical power, while psychological endurance capability becomes the key factor of winning. As times goes on, score change and attitude change of people around and audiences will influence the competition result. Heavy psychological stress of athletes will inevitably influence competition result. This paper carries out quantitative analysis of psychological endurance capability of basketball athletes through document literature and data statistics. Quantitative analysis refers to analysis and comparison of quantitative relation and quantitative change of objects of study to gain research conclusions, usually including trend analysis method and comparative analysis approach. This paper mainly focuses on "Choking" phenomenon which often appears in basketball sport to carry out three specific qualitative analysis experiments. Choking refers to poor performance of basketball athletes under the condition of psychological stress increase.

\section{Experimental design}

\section{Comparison of professional athletes with green hands}

Professional basketball athletes and green hands with general technical level are divided into two groups. Previous normal technical level data of both groups are recorded well beforehand as the control. Different experiment methods are used to test their performance level.

\section{Experimental result statistics}

After members of both groups generate certain psychological stress in the special stage of competition, performance level characteristics of each group are observed to analyze whether "Choking" phenomenon appears. Besides, it is required to analyze and compare whether "Choking" phenomenon changes with time.

\section{Factors influencing psychological endurance capability}

For example, psychological endurance capability can be analyzed and compared through investigating athletes' character, or physical quality and growing environment can be used for the test. 


\section{Experimental hypothesis}

a) The athletes with large strength gap are divided into two groups and they are required to play according to routine method and the specified method. In the experiment, the integral level of green hands is certainly inferior to the group wit high level. When the special method is applied, green hands may not be greatly influenced. Instead, they may perform better than usual. The performance of the group with high level may be influenced.

b) "Choking" phenomenon may become more obvious with the rise in stress. "Choking" phenomenon is not equal to abnormal performance. In basketball competition, such phenomenon is widespread under heavy stress. When the competition starts, psychology of members in both groups does not fluctuate greatly, and "Choking" phenomenon is not obvious. As the competition advances and enters the fierce stage, such phenomenon will become stronger and stronger. Thus, basketball athletes will generate "Choking" phenomenon under heavy stress. This conclusion is established.

c) The relationship between basketball athletes' personal factors (such as character) and their psychological endurance capability is tested. In general, optimistic people have stronger psychological endurance capability, while those with introversion character and weak sense of competition will generate large psychological fluctuation when they encounter tense occasions. Besides, growing environment and family background of basketball athletes can be used for quantitative analysis and comparison of their psychological endurance capability.

\section{Detailed experimental operation and result analysis}

\section{Competition test}

\section{Grouping}

20 boys of a professional high school were chosen for shooting test, with the age ranging from 16 to 18. Their height and weight conformed. 10 boys were professional basketball athletes, and their level was at Level A. The other 10 boys were green hands, and their level was Level B. These boys with different basketball level were divided into Group A and Group B for competition.

\section{Task}

The competition task was shooting. Two comparison tests were conducted for each group, including one normal shooting and one shooting under the specified movement. Members of both groups did not disturb each other. Sufficient time was left for group members to make adjustments.

Method

A common basketball court was chosen. Each member in two groups used 10 balls for shooting test in each test. Firstly, Group A with high level was required to shoot 10 balls within the specified time without any special requirement. During testing members of Group A, members of Group B could not watch and were isolated with members of Group A. After Group A completed the first test, their average hit rate was calculated. Then, 20min test time was given for them. During this period, members of Group B were required to finish shooting in the same way, and their average hit rate was calculated. After 20min, members of Group A challenged the second test. The second test stipulated wrist angle of members of Group A should be $90^{\circ}$ as far as possible during shooting, and the shoulder served as the point of force application for shooting. Then, their shooting state and facial expression change were observed. Meanwhile, their average hit rate was calculated. After the test was finished, $20 \mathrm{~min}$ rest time was given to them. Then, Group B conducted this in the same way. In the last test, no special provision was given to them, and they completed shooting according to their habitual method. Group B finished the third test in the same way, and respective average hit rate was calculated. 
Test result statistics

Group A (professional basketball athletes)

\begin{tabular}{|c|c|c|}
\hline & Average hit rate & Standard deviation \\
\hline First time & 8.8 & 0.40 \\
\hline Second time & 6.9 & 0.56 \\
\hline Third time & 8.6 & 0.49 \\
\hline
\end{tabular}

According to the data in the table, average hit rate difference between the first test and the third test is 0.2 , and there is no significant difference. The hit rate of the second test without external requirement has significant differences with the hit rate of the first test and the third test. This indicates that the performance of Group A with high technical level fluctuates greatly when they are influenced by external provisions. The experimental hypothesis is basically established. Group B (green hands with general level)

\begin{tabular}{|c|c|c|}
\hline & Average hit rate & Standard deviation \\
\hline First time & 7.6 & 0.66 \\
\hline Second time & 7.5 & 0.66 \\
\hline Third time & 7.8 & 0.68 \\
\hline
\end{tabular}

It can be seen from the table that although the performance of Group B in the three tests is inferior to that of Group A on the whole, but the performance does not fluctuate greatly. Even in the second test without special provision, they perform better than professional basketball athletes. This also conforms to experimental hypothesis. Special restriction scarcely influences psychology of green hands.

\section{Statistics of "Choking" phenomenon test}

The three tests of Group A were analyzed. Generally speaking, psychological stress in the first test is smaller than that in the second test. According to the statistical table, standard deviation of Group A in the first test is significantly lower than that in the second test. This indicates that the performance fluctuation is large in the second test without special requirement. This means the members do not perform stably in the second test. In the second test, members' psychological stress is heavy. This shows "Choking" phenomenon becomes more obvious under heavy stress.

\section{Statistical analysis of relationship between basketball athletes' personal factors and psychological endurance capability}

Group A with obvious performance fluctuation was chosen for statistics. The data of 5 members of Group A were drawn at random to analyze and compare their personal factors and shooting performance. (The full score of character value is 10)

Group A

\begin{tabular}{|c|l|l|l|l|l|}
\hline & \multicolumn{1}{|c|}{$\mathrm{a}$} & $\mathrm{b}$ & $\mathrm{c}$ & $\mathrm{d}$ & $\mathrm{e}$ \\
\hline Team awareness & 8.8 & 8.8 & 9.0 & 8.0 & 9.0 \\
\hline Sense of competition & 7.9 & 7.6 & 7.0 & 7.1 & 8.0 \\
\hline Level of optimism & 9.3 & 8.8 & 8.9 & 7.6 & 8.0 \\
\hline Average hit rate & 8.9 & 8.0 & 8.1 & 7.3 & 8.2 \\
\hline Standard deviation & 0.44 & 0.44 & 0.43 & 0.66 & 0.42 \\
\hline
\end{tabular}

According to the general trend of five groups of data, the performance of member a is outstanding, and the performance of member $\mathrm{d}$ falls behind. Character factor indicator and performance level of the other three members conform. The standard deviation of member $d$ is also large. This indicates the performance is not stable. This shows that personal factors have certain influence on the performance. More exactly, personal factors generate influence on psychology.

Each member of a group should have similar strength. The performance of member $\mathrm{d}$ is poor, and the fluctuation is also obvious. This indicates that he should be influenced by psychological problem rather than technical level. In general, the more optimistic one is, the stronger his team awareness, sense of competition and psychological quality will be. Psychological quality of member $\mathrm{d}$ is poor, so external influence may let hi generate psychological stress easily. Especially in the second test, they 
were required to shoot according to the specified movement. On the one hand, they should ensure hitting the target; on the other hand, their movement should be limited. These should have generated certain influence on member $d$ and result in performance instability.

\section{Factors influencing basketball athletes’ psychological endurance capability}

\section{Personal factors}

Every one has different traits of character, and different character will bring different thought. For example, those with anxiety-type personality are very sensitive to the environment. The response of other members and audiences' attitude may generate certain influence on people with such personality in the competition. Such people may lose confidence when audiences have negative response after they lose a ball. Hence, psychological endurance capability of such people is weak. In contemporary mechanism of paying more attention to psychology than skill, some lose the advantage. Those with open personality will not be greatly influenced when they lose one or two balls, and they can treat the competition optimistically. However, the shortcoming is that their awareness of unexpected development is poor. They may have many errors in the competition.

\section{External factors}

For example, expected value of audiences, relatives and friends is too high, which will increase stress on athletes. In the Rio Olympics, numerous audiences stared at table tennis and considered there was no ambiguity about winning the gold medal in this event. Even CCTV also labeled like this, and microblog was also full of such posts. Maybe few people could feel the psychological stress borne by the coach and athletes. The coach Liu Guoliang once said that whenever one more considers we will win in table tennis competition, his stress will increase a little. Although the result is that we win the gold medal, only those who watched the competition on the site would find the process was not so easy. Athletes won the champion step by step, in spite of pains. If they had no strong psychological endurance capability, this result would not be gained. In the first experiment, athletes were required to follow the specified movement. This imposed restrictions on professional athletes who had been accustomed to their own methods. They needed to keep the specified movement under the precondition of avoiding errors. For them, this challenged their psychology. Few could completely overcome this obstacle.

\section{Special time}

Through watching so many basketball competitions, I summarize that the error may be made more easily at the crucial time, such as the game ball. At this moment, psychological stress borne by athletes reaches the peak. If they cannot keep peaceful mentality, unnecessary error will often occur and influence the whole competition result. In such heavy stress, occurrence rate of "Choking" phenomenon is large. When the referee warns the athlete with yellow card or red card, this will also generate psychological influence on the athlete.

\section{Methods to improve athletes’ psychological endurance capability}

\section{Distract attention}

When influenced by external factors, athletes should let them calm down quickly, force them not to think about the thing which has happened, keep positive, breathe deeply, adjust their rhythm and not be influenced by the environment. 


\section{Release stress}

Stress is accumulated gradually. If one cannot reduce stress, he should learn to release the stress, such as pouring out to the parents or trustworthy friends. After pouring out, he will feel relaxed and others will give him some suggestions. This can release the stress to the largest extent.

\section{Improve thought}

Character is a major factor influencing one's psychological endurance capability. Character decides thought. One should not be restricted by rigid thought. For instance, there should be no such idea that "I must win" before the competition. He should tell himself that this is just a competition, and the result is not the most important thing. Enjoying the competition process is more important. It does not matter to fail in one competition, and there will be many competitions in the future. One failure cannot explain anything. If athletes think in this way, they will feel relaxed and face the completion with usual mind.

\section{Broaden horizon}

Athletes should travel often and view something new. In this way, they will broaden their horizon and become optimistic. One or two competitions will not restrict one's thought. They should relieve stress and release themselves.

\section{References}

[1] Wang Ruiyuan, Exercise physiology. Beijing: Peoples Sports Publishing House, 2002.

[2] Zhang Liwei, Emotional factor structure, self-statement evaluation and notice before competition. Beijing Sport University Press, 2001, 29.

[3] Ma Qiwei, Sports physiology. Beijing: Higher Education Press, 2001.

[4] Wang Peng, Analysis of athletes’ psychological training. Liaoning Sport Science and Technology, 2005(6): 109.

[5] Xia Siyong, Contemporary sports science. Southwest China Normal University Press, 2006(4): 16-20. 